\section{BMJ Open} Ophthalmology

\title{
Effects of intraocular lens glistenings on visual function: a prospective study and presentation of a new glistenings grading methodology
}

\author{
Nick Stanojcic, ${ }^{\oplus 1}$ David P S O'Brart, ${ }^{1}$ Nick Maycock, ${ }^{2}$ Chistopher C Hull ${ }^{3}$
}

To cite: Stanojcic N, O'Brart DPS, Maycock N, et al. Effects of intraocular lens glistenings on visual function: a prospective study and presentation of a new glistenings grading methodology. BMJ Open Ophthalmology 2019;4:e000266. doi:10.1136/ bmjophth-2018-000266

23rd European Society of Cataract and Refractive Surgeons (ESCRS) Winter Meeting, Athens, Greece, February 2019

Received 20 December 2018 Revised 19 February 2019 Accepted 20 February 2019
Check for updates

(c) Author(s) (or their employer(s)) 2019. Re-use permitted under CC BY-NC. No commercial re-use. See rights and permissions. Published by BMJ.

${ }^{1}$ Department of Ophthalmology, St Thomas' Hospital, London, UK 'Department of Ophthalmology, Queen Alexandra Hospital, Portsmouth, UK

${ }^{3}$ Centre for Applied Vision Research, School of Health Sciences, City, University of London, London, UK

Correspondence to Mr Nick Stanojcic; nstanojcic@ doctors.org.uk

\section{ABSTRACT}

Objective To investigate the effect of intraocular lens (IOL) glistenings on visual performance and evaluate a new glistenings grading methodology.

Methods and Analysis Thirty-four patients (34 eyes) were recruited. Corrected distance visual acuity (CDVA), mesopic gap acuity (MGA), functional contrast sensitivity (FCS) and forward light scatter were measured (Advanced Vision and Optometric Tests, City Occupational, London, UK). The IOL centre was imaged and glistenings density graded by three observers using the Miyata scale and a new system. Inter-rater reliability, association between the two grading scales, and correlations between glistenings grades and visual performance parameters were evaluated.

Results The intraclass correlation coefficient between graders for the new grading system was 0.769 (95\% Confidence Interval [Cl] 0.636 to 0.868 ). There was a significant association between the Miyata scale and the new grading system for all graders $\left(r_{s}=0.533-0.895\right.$, $\mathrm{p} \leq 0.001)$. There was no association between CDVA or MGA and glistenings grade $\left(r_{s}=-0.098, p=0.583\right.$ and $r_{s}=0.171, p=0.359$, respectively). There was no association between FCS at mesopic light levels and glistenings grade $(r=-0.032, p=0.864)$, or the straylight parameter and glistenings grade $\left(r_{s}=0.021, p=0.916\right)$. No association was found between the integrated straylight parameter and glistenings grade $\left(r_{s}=0.078, p=0.701\right)$.

Conclusion The new glistenings grading scale was highly reproducible. In this cohort, glistenings in the same hydrophobic acrylic IOL after cataract surgery were not associated with changes in visual function, as assessed by a series of tests not previously used in glistenings research.

\section{INTRODUCTION}

Glistenings are vacuoles that can develop within intraocular lenses (IOLs) implanted as part of cataract surgery. They occur in all IOL materials but are mostly associated with hydrophobic acrylic IOLs. ${ }^{1-3}$ Glistenings form when water permeates through microchannels within the material to create small fluid-filled inclusions that are up to $30 \mu \mathrm{m}$ in size. $^{4-9}$

\section{Key messages}
What is already known about this subject?
- There is a limited amount of evidence that intraoc- ular lens (IOL) glistenings may have an effect on vi- sual function and in particular high spatial frequency contrast sensitivity.
> Previously described subjective grading scales of IOL glistenings in vivo vary in terms of defining the IOL area/volume under investigation, specifying im- aging equipment settings and ambient illumination levels.

\section{What are the new findings?}

- We have described a new, highly reproducible grading methodology with optimum equipment settings specifically developed for the purpose of grading glistenings and with carefully controlled ambient illumination.

- In this cohort, glistenings in the same hydrophobic acrylic IOL after cataract surgery were not associated with visual function parameters, including visual acuity, positive and negative contrast sensitivity at 10 cycles per degree, and forward light scatter.

\section{How might these results change the focus of} research or clinical practice?

- The detailed method for evaluating IOLs in vivo and the new grading scale may help standardise IOL glistenings grading.

The new finer, highly reproducible grading scale may be more suitable for comparing modern IOLs with small amounts of glistenings as well as IOLs with relatively large number of glistenings.

While most previous studies have demonstrated no significant effect of glistenings on vision, ${ }^{10-16}$ a few have found that high numbers of such vacuoles within IOLs can impair visual performance, ${ }^{59}$ especially high spatial frequency contrast sensitivity. ${ }^{17-20}$

Labuz et $a l^{6}$ published an in vitro model for predicting the straylight parameter from the density of glistenings. They found that 'large numbers' of glistenings were needed to cause straylight levels that might lead to 
glare-related visual problems. However, in vivo the issue of accurately determining the numbers and density of IOL glistenings remains a challenge, especially with regard to eye/patient macromovements and micromovements during imaging, variation of light entering the eye with natural physiological variations and anomalies, and the limitations of current clinical diagnostic imaging technologies. As a result, it is difficult to test the precise relationship between glistenings density and visual performance in vivo.

Previously published in vivo glistenings grading systems have typically used estimating or counting methodologies with a single examiner, differing reported slit-lamp (SL) magnifications $\left(16 \times,{ }^{5} 1721,25 x^{17}, 40 \times\right),{ }^{18}$ ordinal scale ranges ([0 to 2$],{ }^{11}{ }^{18}[0$ to 3$],{ }^{217}[0$ to $3+],{ }^{21}[0$ to +4$],{ }^{20}$ [trace to $\left.3+\right]^{19}$, [trace to 4$\left.]\right),{ }^{522}$ and different highest grade cut-offs $\left([>50],{ }^{21}[40\right.$ 'per field' $],{ }^{52}[150$ per $\left.\left.\mathrm{mm}^{2}\right]^{18},\left[200 \text { per } \mathrm{mm}^{2}\right]^{7}\right)$. Previous studies that have used subjective evaluation and grading of glistenings at the SL or from SL-derived photographs have not defined the region of the IOL being studied, specified illuminance levels or image capture parameters. ${ }^{25} 1117192122$ Moreover, correlation between glistenings grading at the SL and that of IOL images has been found to be only moderate. ${ }^{22}$

To try and more accurately describe the relationship between IOL glistenings and visual performance, in this study we developed a new, precisely defined, subjective, finer, zonal grading system based on high-quality colour digital SL images taken with a described, fixed protocol and using three graders. Agreement of glistenings grading between the graders was analysed and the new scale compared with a previously described and published grading system in widespread usage. ${ }^{7}$ To investigate the association between glistenings and visual performance, we employed a range of tests at photopic and mesopic light levels, including logarithm of the minimum angle of resolution ( $\log$ MAR) visual acuity, straylight measurements, gap acuity and functional contrast sensitivity (FCS).

\section{MATERIALS AND METHODS}

Thirty-four patients were recruited between September 2017 and February 2018. All had undergone uncomplicated cataract surgery with phacoemulsification and were implanted with monofocal, spherical, hydrophobic acrylic IOLs (Alcon AcrySof SA60AT). Given the novel methodologies for visual testing employed in this investigation, it was designed as a pilot study to help generate results that could power a future main study. Inclusion criteria were age between 18 and 100 with corrected distance visual acuity (CDVA) in the study eye equal to or better than $0.2 \log$ MAR. Prior to study entry, all subjects underwent an ophthalmic examination, including mydriatic funduscopy and, where appropriate, macular optical coherence tomography scans. Exclusion criteria included signs of dry eye, corneal opacities, failure of pupillary mydriasis beyond $5.0 \mathrm{~mm}$, posterior capsule opacification, glaucoma, age-related macular degeneration, retinal vascular disorders, previous retinal detachments, neuro-ophthalmological conditions, inherited retinal disorders or pathology, previous strabismus surgery or history of amblyopia, previous transient ischaemic attack, cerebrovascular attack, or other vaso-occlusive disease or epilepsy.

\section{Assessment of glistenings}

Following pupillary mydriasis, with tropicamide $1 \%$ and phenylephrine $2.5 \%$, central images of the IOLs were taken with a 5-megapixel digital camera (Topcon DC-4, Topcon, Tokyo, Japan) mounted on an SL (Topcon SL-701, Topcon). To obtain the best images of IOL glistenings, prior to starting this study, one author (NS) systematically evaluated different SL and digital camera settings to optimise quality of images.

The same mesopic conditions in the examination room were maintained, with only the assessor's screen on minimal brightness turned away from the patient (ambient illuminance on the SL table did not exceed 0.3 lux). A vertical slit beam of $10.0 \mathrm{~mm}$ by $2.0 \mathrm{~mm}$ at an angle of $40^{\circ}$ and $16 \times$ objective magnification was used with the SL set to maximum brightness to illuminate the centre of the IOL within the pupil. For the Topcon DC-4 camera, an ISO of 800 was used with a shutter speed of $1 / 30 \mathrm{~s}$, a sharpness of ' +32 ' (default), a denoising of ' 0 ' (default), a contrast 'of 50' (default) and the 'auto-brightness' setting at 'off'.

The best of five colour digital SL images from each study eye (one per patient) was selected for analysis. Each was processed by fitting the pupil, identifying its centre and overlaying a $5.0 \mathrm{~mm}$ by $1.0 \mathrm{~mm}$ grid divided into 1.0 $\mathrm{mm}^{2}$ areas (figure 1 ).

Three ophthalmologists (NS, NM and DPSO'B) assessed and graded the digital images independently and within each of the five defined $1.0 \mathrm{~mm}^{2}$ grid squares $(\mathrm{Z}+2$, $\mathrm{Z}+1, \mathrm{Z} 0, \mathrm{Z}-1, \mathrm{Z}-2)$ by counting the number of glistenings they could identify within each separate area. Graders disregarded artefacts such as anterior or posterior IOL surface particulates (eg, pigment), IOL scratches and scuff marks (eg, from the IOL loading device or forceps), posterior capsule irregularities, and vitreous floaters. To reduce assessor bias, image strips were randomised and presented to the graders for evaluation in PowerPoint (Microsoft, Washington, USA) by a fellow researcher not involved in grading $(\mathrm{CH})$.

Grades of glistening density were then assigned to each $1.0 \mathrm{~mm}^{2}$ area according to the following 8-point ordinal scale: grade $0=$ no visible glistenings, grade $1=1-10$ per $\mathrm{mm}^{2}$, grade $2=11-20$ per $\mathrm{mm}^{2}$, grade $3=21-30$ per $\mathrm{mm}^{2}$, grade $4=31-40$ per $\mathrm{mm}^{2}$, grade $5=41-50$ per $\mathrm{mm}^{2}$, grade $6=51-60$ per $\mathrm{mm}^{2}$ and grade $7>61$ glistenings per $\mathrm{mm}^{2}$.

In addition, the central three zones for the new scale were also graded. This was undertaken to see if it provided greater reproducibility. In addition, a $3 \mathrm{~mm}$ pupil is the average pupil size under photopic conditions for most individuals over the age of $70 .^{23}$ 


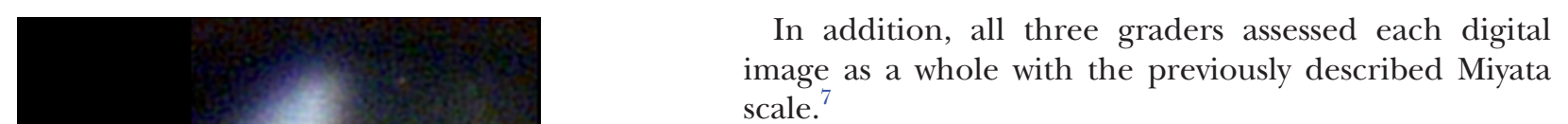
scale. $^{7}$

Agreement of glistenings grading between the examiners and association between the two scales were evaluated.

\section{Visual testing}

Photopic visual acuity was assessed using an Early Treatment Diabetic Retinopathy Study backlit chart at $4 \mathrm{~m}$ (Precision Vision, Illinois, USA).

Advanced Vision and Optometric Tests (AVOT, City Occupational, London, UK) were used under mesopic conditions where ambient illuminance on the display surface did not exceed 0.3 lux and background display screen luminance was $1 \mathrm{Cd} \mathrm{m}^{-2}$ :

1. Gap acuity using a Landolt ring for both positive and negative contrast at a viewing distance of $3 \mathrm{~m}$. The orientation of the Landolt ring was restricted to four locations and the subject pressed one of the four buttons available on a bespoke keypad to indicate the location of the gap.

2. FCS using a Landolt ring (as for mesopic gap acuity) with a 3' gap for both positive and negative contrast at a viewing distance of $3 \mathrm{~m}$. A gap size of 3' (Landolt ring size $15^{\prime}$ or 10 cycles per degree) was employed to avoid eyestrain and to minimise the effects of microfluctuations of accommodation. ${ }^{24}$ FCS is a measure of contrast sensitivity that has been found to be relevant in occupational environments. ${ }^{25} 26$

3. Forward light scatter test. The forward light scatter test implemented in the AVOT system used a flicker cancellation method. ${ }^{27} 28$ It employed a single ring of fixed size designed to produce specified luminance levels in the plane of the pupil from a viewing distance of $70 \mathrm{~cm}$. The participant used a single button on a bespoke keypad to neutralise the flicker that appeared on the screen. Both 'low threshold' and 'high threshold' were evaluated. The software calculated the straylight parameter and integrated straylight parameter.

The association between glistenings grade and all the tested visual performance parameters described above were tested.

\section{Statistical methods}

One eye was randomly selected for each participant. Data organisation and descriptive statistics were handled with Excel 2013 (Microsoft Corporation, WA, USA), with further statistical analyses performed with Minitab V.14 (Minitab, Pennsylvania, USA).

The subjective glistenings grade was calculated as the median of all five zones for each grader since the data were skewed and the grades were on an ordinal scale. The overall grade used in the analysis of association with visual performance parameters was taken as the median of all three graders. Inter-rater reliability was assessed using the intraclass correlation coefficient. Agreement between grades for the median of the central three
Figure 1 Slit-lamp image of pupillary centre (slit-lamp beam width and height setting: $2.0 \mathrm{~mm}$ and $10.0 \mathrm{~mm}$, respectively) with an overlay of a $5.0 \mathrm{~mm}$ by $1.0 \mathrm{~mm}$ grid, which was divided into $1.0 \mathrm{~mm}$ by $1.0 \mathrm{~mm}$ areas, with the central area bisecting the pupillary centre. 
zones and the median of all five zones was assessed using weighted kappa. Association between the new grading system and the Miyata scale was assessed with a $\chi^{2}$ test for independence. Associations between glistenings grade and visual performance parameters were measured using Spearman's rho. The significance level was taken to be $5 \%$ in all cases.

\section{RESULTS}

\section{Patient demographics}

The mean subject age was 72.9 \pm 5.1 years (Standard Deviation [SD], range $63-85$ years). Eleven subjects (32\%) were female and $23(68 \%)$ were male. Study participants had undergone cataract surgery between 5 and 66 months previously (median 14 months), with $50 \%$ of the participants having had surgery $9.75-14.25$ months previously. Random selection resulted in $24(71 \%)$ right and $10(29 \%)$ left eyes.

\section{Glistening grades}

Of the 34 IOLs evaluated with the new grading scale, 20 $(59 \%)$ had grade 1 glistenings, $8(23 \%)$ grade $2,3(9 \%)$ grade 3,1 (3\%) grade 4 and $2(6 \%)$ grade 5 when considering all five zones.

With regard to the central three zones, $17(50 \%)$ had grade $1,9(26 \%)$ grade $2,5(15 \%)$ grade $3,1(3 \%)$ grade 4, 1 (3\%) grade 5 and 1 (3\%) grade 7 glistenings.

Using the median grade for all three graders with the Miyata scale, of the 34 eyes, 1 (3\%) was grade $0,27(79 \%)$ grade $1,4(12 \%)$ grade 2 and $2(6 \%)$ grade 3.

\section{Reliability and reproducibility of the new grading system}

The intraclass correlation coefficient, for the median of the grade for all five zones, was 0.769 (95\% CI 0.636 to 0.868 ), indicating a 'good' level of agreement between graders. The intraclass correlation coefficient for the median of the grade of the central three zones was slightly higher at 0.813 (95\% CI 0.696 to 0.895 ). Intraobserver agreement between the grades for all five zones versus the central three zones for the three graders was assessed using quadratically weighted kappa. The values of 0.948 , 0.875 and 0.854 , respectively, demonstrated 'very good' levels of agreement. The association between the Miyata and the new grades (median of the five zones) for all three graders was statistically significant $(\mathrm{p} \leq 0.003)$.

\section{Association between subjective assessment of glistenings and visual function logMAR distance acuity}

For the 34 study eyes, the mean logMAR uncorrected distance acuity was $0.06 \pm 0.12$ (SD) (median 0.01 , range -0.1 to 0.5 ). The mean CDVA was $-0.04 \pm 0.07$ (SD) (median -0.06 , range -0.2 to 0.18 ).

There was no association between glistenings grade and high-contrast CDVA when the median grade for the five zones was considered $\left(\mathrm{r}_{\mathrm{s}}=-0.098, \mathrm{p}=0.583\right)$ nor when the central three zones were considered $\left(\mathrm{r}_{\mathrm{s}}=-0.045, \mathrm{p}=0.80\right)$.

Gap acuity (minutes of arc)

At positive target contrast, there was no association between gap acuity and grade of glistenings when the median grade for the five zones was considered $\left(\mathrm{r}_{\mathrm{s}}=0.171\right.$, $\mathrm{p}=0.359)$ nor when median grade for the central three zones was considered $\left(\mathrm{r}_{\mathrm{s}}=0.137, \mathrm{p}=0.461\right)$. Similarly, at negative target contrast, there was no association between gap acuity and grade of glistenings when the median grade for the five zones was considered $\left(r_{s}=0.009\right.$, $\mathrm{p}=0.962$ ) nor when the median grade for the central three zones was considered $\left(r_{s}=0.007, p=0.968\right)$. There was no statistically significant difference $(\mathrm{p}=0.691)$ between positive and negative mesopic gap acuity. Three of 34 (9\%) subjects could not complete the mesopic gap acuity test.

\section{Functional contrast sensitivity}

At positive target contrast, there was no association between FCS and grade of glistenings regardless of whether the median grade for the five zones was considered $\left(r_{s}=-0.032, p=0.864\right)$ or the median for the central three zones was considered $\left(\mathrm{r}_{\mathrm{s}}=-0.009, \mathrm{p}=0.962\right)$.

\section{Straylight}

There was no association between the straylight parameter, $\mathrm{k}$, and the median grade of glistenings for neither the five zones $\left(\mathrm{r}_{\mathrm{s}}=0.021, \mathrm{p}=0.916\right)$ nor for the central three zones $\left(r_{s}=0.050, p=0.803\right)$. Similarly, there was no association between the integrated straylight parameter, $\mathrm{k}$, and the median grade of glistenings for neither the five zones $\left(\mathrm{r}_{\mathrm{s}}=0.078, \mathrm{p}=0.701\right)$ nor the central three zones $\left(r_{s}=0.088, p=0.663\right)$. Seven out of $34(21 \%)$ subjects could not complete the light scatter test.

\section{DISCUSSION}

The results of the present study add to the evidence that glistenings, unless possibly present in extremely large amounts, have a minimal effect on visual performance in vivo. Our results add new knowledge because they confirm the results of some previous studies, ${ }^{10-16}$ but used novel visual assessment methods and a novel grading system using three graders and strictly controlled imaging and ambient illuminance parameters.

Our results demonstrate good agreement between graders and a high level of correlation with an existing scale. $^{7}$ Our grading system offers benefits over that described by Miyata ${ }^{7}$ as it has precisely defined imaging and ambient illuminance parameters, as well as definition of the region of the IOL being analysed, which can be reproduced by other clinical investigators. Indeed, we find the Miyata system was somewhat confusing as it originally described the grading scale in three dimensions ${ }^{29}$ or per $\mathrm{mm}^{3}$ but later ${ }^{7}$ referenced the scale to a two-dimensional image or per $\mathrm{mm}^{2}$. This might explain why the high number of glistenings in the highest Miyata grade does not seem to correspond to the number of glistenings observed in the actual reference images. ${ }^{729}$

Glistenings are small fluid-filled inclusions, up to $30 \mu \mathrm{m}$ in size. ${ }^{4-9}$ Detection of glistenings is dependent on their luminance contrast and not size. Therefore, provided there is enough light reflected they will be seen, although some appear fainter than others. Rather than size, one 
of the main difficulties is to resolve multiple glistenings which are close together. To help overcome this, our digital image strips were divided into five separate $1 \mathrm{~mm}^{2}$ areas, centred on the pupil and presented at full screen size on a computer monitor for grading. Three graders performed the grading, allowing us to evaluate both the interobserver and intraobserver reliability of our method, which was very high. Such accuracy of a grading system based on counting is not possible, with observers counting at the SL; this is due to factors such as patient discomfort and movement due to bright light and lack of reference points, and the large numbers of glistenings requiring counting in some cases.

As discussed above, unlike previous studies that used digital images and counting methodologies, ${ }^{9} 12131822$ we systematically analysed and optimised the parameters of a commercially available SL-based digital imaging system under controlled conditions to reduce artefacts and maximise the quality of glistenings images taken in vivo. In addition, we identified, imaged and graded the same central pupillary area of each IOL and divided this area for analysis into five $1 \mathrm{~mm}^{2}$ areas in which the numbers of glistenings could be reliably identified and counted. Finally, to provide a further level of consistency, we excluded any eyes and patients for analysis with ocular and/or neurological comorbidities that might affect visual performance and all participants had the same design of IOL. Using our system, we analysed both the grades from all five $1 \mathrm{~mm}^{2}$ zones and the central three zones. Using the central three zones may be more relevant to the photopic pupil size of those aged 70 or above ab $^{23}$ and could avoid artefacts seen near the edge of the pupil (eg, reflections or opacity from the anterior capsule). There was good agreement and little difference in the results for grades using all five zones and the central three zones, suggesting we can simplify our grading protocol.

The series of visual function tests from the AVOT system have not been used in studies of glistenings before but have been used in other vision research. ${ }^{30}$ FCS has been shown to be a more sensitive indicator of changes in the quality of the retinal image caused by small residual refractive errors, higher order aberrations and/ or scattered light. As the FCS test provides a measure of contrast sensitivity that has been shown to be relevant in occupational environments, ${ }^{25}$ it seems appropriate for evaluating how IOL glistenings can affect image changes caused by increased scattered light and aberrations and for evaluating the 'real-life' visual effects of IOL glistenings.

There are limitations to our study. We evaluated only the number of glistenings, while their sizeand surface portion $^{69}$ might also affect visual function. The glistenings grades in our study participants had a narrow IQR probably because the time post cataract surgery was closely grouped around a median of 14 months and/or due to manufacturing changes introduced in the past few years $^{31}$ in the IOL implanted in these patients to limit glistenings. Our results, however, included patients with what would have been described by a previous grading system $^{5}$ as mild to severe levels of glistenings. Indeed, if we applied the grading scale as described by Christiansen et $a \tilde{l}$ to our cohort, then $24 \%$ of our cases ( 8 out of 34 eyes) would have 'severe glistenings' (in these eyes at least one of the five squares was graded as our grade ' 5 ' by at least one grader). We believe that our scale by virtue of its eight grading steps allows more detailed grading of the highest glistenings densities than other grading systems, ${ }^{521} 22$ which tend to group such changes together. Moreover, by allowing finer stratification of glistenings densities that may not have been isolated by other grading scales, it may be more suitable for comparing modern IOLs with fewer glistenings.

It is difficult to compare our grading system with the semiautomated counting methods, ${ }^{9} 121318$ which are largely operator-dependent and may detect image artefacts as well as true glistenings. It has been postulated that Scheimpflug images might be used to detect glistenings in vivo. ${ }^{32}$ However, it appears that this method is not suitable for such evaluation as it cannot distinguish between light scatter due to glistenings and that from other optical changes, such as aqueous-IOL interface, posterior capsule or debris on the IOL surface. ${ }^{33}$ Indeed, Biwer et al found that a Scheimpflug device did not provide images of required resolution to perform automated counting of separate glistenings. ${ }^{22}$ This has also been our experience with a Scheimpflug device (Pentacam HR; Oculus Optikgeräte, Wetzlar, Germany).

Colin $e a^{11}$ reported that approximately a third of their AcrySof IOL cohort had no glistenings, a third had grade 1 glistenings and about a third had grade 2 glistenings (the most severe grade in their system). Similarly, Christiansen et al found that $65 \%$ of their AcrySof patients had 'trace' glistenings, while grades ' $1+$ ' and grade '2+' accounted for $25 \%$ and grades ' $3+$ ' and ' $4+$ ' accounted for $5 \%$ each. Our results, where, for the central three zones, $50 \%$ had grade 1, $41 \%$ had grades $2-3$ and $9 \%$ had grade 4 or above, appear to show a similar range of IOL glistenings.

Three of the $34(9 \%)$ of our subjects could not complete the FCS test, and 7 of $34(21 \%)$ could not complete the light scatter test. The AVOT tests we used are essentially psychophysical tests requiring high levels of cognitive functioning. The limitation of the FCS test is its length of time if a participant is not able to resolve the 3' Landolt ring gap accurately, and the light scatter test is limited because it cannot test participants with very low flicker sensitivity. These limitations of test methods leading to some incomplete data are also found in previously reported studies, using other assessment methodologies. Colin and Orignac ${ }^{13}$ reported that in only 53 of 97 cases (54\%) could valid measurements be produced when they measured intraocular light scatter in their cohort of patients with glistenings, with the C-Quant test (Oculus Optikgeräte, Wetzlar, Germany).

In common with many previous studies, we have demonstrated no significant effect of glistenings on visual 
function as measured by a series of novel tests under strictly controlled conditions. In order to standardise glistenings grading in vivo, we developed a new protocol that uses three graders and that images IOL with optimised digital camera parameters under controlled ambient illuminance, which may be a useful tool for future research.

Contributors NS: conception, design, collecting data, analysis and interpretation of data, drafting the article, revising the article. DPSO'B: conception, design, analysis and interpretation of data, drafting the article, revising the article. NM: conception, design, data analysis, drafting the article, revising the article. $\mathrm{CCH}$ : conception, design, analysis and interpretation of data, drafting the article, revising the article.

Funding The authors have not declared a specific grant for this research from any funding agency in the public, commercial or not-for-profit sectors.

Competing interests DPSO'B reports grants and personal fees from Alcon, outside the submitted work. CCH reports grants from Alcon, outside the submitted work.

Patient consent for publication Not required.

Ethics approval This study was approved by London - Bloomsbury Research Ethics Committee. This research conformed to the tenets of the Declaration of Helsinki.

Provenance and peer review Not commissioned; externally peer reviewed.

Open access This is an open access article distributed in accordance with the Creative Commons Attribution Non Commercial (CC BY-NC 4.0) license, which permits others to distribute, remix, adapt, build upon this work non-commercially, and license their derivative works on different terms, provided the original work is properly cited, appropriate credit is given, any changes made indicated, and the use is non-commercial. See: http://creativecommons.org/licenses/by-nc/4.0/.

\section{REFERENCES}

1. Werner L. Glistenings and surface light scattering in intraocular lenses. J Cataract Refract Surg 2010;36:1398-420.

2. Tognetto D, Toto L, Sanguinetti G, et al. Glistenings in foldable intraocular lenses. J Cataract Refract Surg 2002;28:1211-6.

3. Miyata A, Yaguchi S. Equilibrium water content and glistenings in acrylic intraocular lenses. J Cataract Refract Surg 2004;30:1768-72.

4. Kato K, Nishida M, Yamane $\mathrm{H}$, et al. Glistening formation in an Acrysof lens initiated by spinodal decomposition of the polymer network by temperature change. J Cataract Refract Surg 2001;27:1493-8.

5. Christiansen G, Durcan FJ, Olson RJ, et al. Glistenings in the ACRYSOF intraocular lens: pilot study. J Cataract Refract Surg 2001;27:728-33.

6. Łabuz G, Reus NJ, van den Berg TJTP. Straylight from glistenings in intraocular lenses: In vitro study. J Cataract Refract Surg 2017;43:102-8.

7. Miyata A,, Uchida N, Nakajama N, et al. Clinical and experimental observation of Glistening in acrylic intraocular lenses. Jpn J Ophthalmol 2001;45:564-9.

8. Dogru M, Tetsumoto K, Tagami Y, et al. Optical and atomic force microscopy of an explanted ACRYSOF intraocular lens with glistenings. J Cataract Refract Surg 2000;26:571-5.

9. Henriksen BS, Kinard K, Olson RJ. Effect of intraocular lens glistening size on visual quality. J Cataract Refract Surg 2015;41:1190-8.

10. Mönestam E, Behndig A. Impact on visual function from light scattering and glistenings in intraocular lenses, a long-term study. Acta Ophthalmol 2011;89:724-8.

11. Colin J, Praud D, Touboul D, et al. Incidence of glistenings with the latest generation of yellow-tinted hydrophobic acrylic intraocular lenses. J Cataract Refract Surg 2012;38:1140-6.
12. Waite A, Faulkner N, Olson RJ. Glistenings in the single-piece, hydrophobic, acrylic intraocular lenses. Am J Ophthalmol 2007;144:143-4.

13. Colin J, Orignac I. Glistenings on intraocular lenses in healthy eyes: effects and associations. J Refract Surg 2011;27:869-75.

14. Hayashi K, Hirata A, Yoshida M, et al. Long-term effect of surface light scattering and glistenings of intraocular lenses on visual function. Am J Ophthalmol 2012;154:240-51.

15. Chang A, Behndig A, Rønbeck $M$, et al. Comparison of posterior capsule opacification and glistenings with 2 hydrophobic acrylic intraocular lenses: 5- to 7-year follow-up. J Cataract Refract Surg 2013;39:694-8.

16. Chang A, Kugelberg M. Glistenings 9 years after phacoemulsification in hydrophobic and hydrophilic acrylic intraocular lenses. J Cataract Refract Surg 2015;41:1199-204.

17. Xi L, Liu Y, Zhao F, et al. Analysis of glistenings in hydrophobic acrylic intraocular lenses on visual performance. Int $J$ Ophthalmol 2014;7:446-51.

18. Schweitzer C, Orignac I, Praud D, et al. Glistening in glaucomatous eyes: visual performances and risk factors. Acta Ophthalmol 2014;92:529-34.

19. Dhaliwal DK, Mamalis N, Olson RJ, et al. Visual significance of glistenings seen in the ACRYSOF intraocular lens. J Cataract Refract Surg 1996;22:452-7.

20. Gunenc U, Oner FH, Tongal S, et al. Effects on visual function of glistenings and folding marks in Acrysof intraocular lenses. J Cataract Refract Surg 2001;27:1611-4.

21. Wilkins E, Olson RJ. Glistenings with long-term follow-up of the surgidev B20/20 polymethylmethacrylate intraocular lens. Am J Ophthalmol 2001;132:783-5.

22. Biwer H, Schuber E, Honig M, et al. Objective classification of glistenings in implanted intraocular lenses using scheimpflug tomography. J Cataract Refract Surg 2015;41:2644-51.

23. Winn B, Whitaker D, Elliott DB, et al. Factors affecting light-adapted pupil size in normal human subjects. Invest Ophthalmol Vis Sci 1994;35:1132-7.

24. Barbur JL, Stockman A. Photopic, Mesopic and Scotopic Vision and Changes in Visual Performance. In: Dartt D, Besharse JC, Dana R, eds. Encyclopedia of the Eye. Oxford, UK: Elsevier, 2010: 323-31.

25. Chisholm CM, Barbur JL, Edgar DF, et al. The effect of excimer laser refractive surgery on visual performance. Invest Ophthalmol Vis Sci 2000;41:S462.

26. Chisholm CM, Evans ADB, Harlow JA, et al. New test to assess Pilot's vision following refractive surgery. Aviat Space Environ Med 2003;74:551-9.

27. van den Berg TJTP, Spekreijse H. Measurement of the straylight function of the eye in cataract and other optical media disturbances by means of a direct compensation method. Invest Ophthalmol Vis Sci 1987;28:397.

28. Hennelly ML. The effect of age on the light scattering characteristics of the eye. Ophthal Physiol Opt 1998;18:197-203.

29. Miyata A, Suzuki K, Boku C, et al. Glistening particles in the implanted acrylic intraocular lens. Jpn J Clin Ophthalmol 1997;51:729-32.

30. Gillespie-Gallery H, Konstantakopoulou E, Harlow JA, et al. Capturing age-related changes in functional contrast sensitivity with decreasing light levels in monocular and binocular vision. Invest Ophthalmol Vis Sci 2013;54:6093-103.

31. Thomes BE, Callaghan TA. Evaluation of in vitro glistening formation in hydrophobic acrylic intraocular lenses. Clin Ophthalmol 2013;7:1529-34.

32. Behndig A, Mönestam E. Quantification of glistenings in intraocular lenses using scheimpflug photography. J Cataract Refract Surg 2009;35:14-17.

33. Mackool RJ, Colin J. Limitations of scheimpflug photography in quantifying glistenings [letter]. J Cataract Refract Surg 2009;35:1480-1. 